\title{
Advanced simulation study on bunch gap transient effect
}

\author{
Tetsuya Kobayashi and Kazunori Akai \\ KEK, Oho 1-1 Tsukuba, Ibaraki, Japan
}

(Received 21 December 2015; published 9 June 2016)

\begin{abstract}
Bunch phase shift along the train due to a bunch gap transient is a concern in high-current colliders. In KEKB operation, the measured phase shift along the train agreed well with a simulation and a simple analytical form in most part of the train. However, a rapid phase change was observed at the leading part of the train, which was not predicted by the simulation or by the analytical form. In order to understand the cause of this observation, we have developed an advanced simulation, which treats the transient loading in each of the cavities of the three-cavity system of the accelerator resonantly coupled with energy storage (ARES) instead of the equivalent single cavities used in the previous simulation, operating in the accelerating mode. In this paper, we show that the new simulation reproduces the observation, and clarify that the rapid phase change at the leading part of the train is caused by a transient loading in the three-cavity system of ARES. KEKB is being upgraded to SuperKEKB, which is aiming at 40 times higher luminosity than KEKB. The gap transient in SuperKEKB is investigated using the new simulation, and the result shows that the rapid phase change at the leading part of the train is much larger due to higher beam currents. We will also present measures to mitigate possible luminosity reduction or beam performance deterioration due to the rapid phase change caused by the gap transient.
\end{abstract}

DOI: 10.1103/PhysRevAccelBeams.19.062001

\section{INTRODUCTION}

In a high-current multibunch storage ring, a gap with empty buckets is introduced in the bunch train to allow for the rise time of a beam abort kicker. The gap is also effective in clearing ions in electron storage rings. The gap, however, modulates the amplitude and phase of the accelerating cavity field. Consequently, the longitudinal synchronous position is shifted bunch-by-bunch along the train, which shifts the collision point of each bunch in a collider. This can result in luminosity degradation or even degradation of the beam performance due to beam-beam effects.

KEKB is an asymmetric energy collider consisting of an $8 \mathrm{GeV}$ electron high-energy ring (HER) and a $3.5 \mathrm{GeV}$ positron low-energy ring (LER), which was operated from 1998 to 2010 [1]. The record luminosity of KEKB was $2.11 \times 10^{34} \mathrm{~cm}^{-2} \mathrm{~s}^{-1}$ and the collider contributed significantly to the study of $C P$-violation physics. The design parameters of KEKB relating to the rf system are shown in Table I. To increase the luminosity, a high-current beam is needed in both rings, which is accomplished by filling particle bunches into a number of buckets. One serious concern for high-current storage rings is the coupled-bunch instability caused by the accelerating mode of the cavities. This issue arises from the large detuning of the resonant

Published by the American Physical Society under the terms of the Creative Commons Attribution 3.0 License. Further distribution of this work must maintain attribution to the author(s) and the published article's title, journal citation, and DOI. frequency of the cavities that is needed to compensate for the reactive component of the beam-loading [2]. The detuning frequency can be comparable to the revolution frequency for a large circumference ring, which results in a very high impedance driving this instability. Two types of cavities that mitigate this problem are used in KEKB $[3,4]$ : one is the ARES normal conducting three-cavity system $[5,6]$ and the other is the superconducting cavities (SCCs) $[7,8]$. The detuning frequency of these cavities is reduced owing to the high stored energy in these cavities.

The ARES is a cavity unit specialized for KEKB. It consists of a three-cavity system operated in the $\pi / 2$ mode: the accelerating (A-)cavity is coupled to a storage (S-) cavity via a coupling (C-)cavity as shown in Fig. 1 [9]. The A-cavity is structured to damp higher-order modes (HOM). The C-cavity is equipped with a damper to damp parasitic 0 and $\pi$-modes associated with the accelerating mode. Because the $\pi / 2$ mode produces a field in the C-cavity that is negligibly small compared to the field in the A- and S-cavities (see the coupled pendulum model in Fig. 1), the $\pi / 2$ mode has a high Q-value even with a C-cavity with a very low $\mathrm{Q}$-value of about 100. For details regarding the ARES cavity, see Refs. [5,6]. Both ARES and SCC were used in HER with an appropriate relative phase to assign a heavier beam loading to ARES and higher cavity voltage to SCC [3]. In LER, where a higher beam current is stored than in HER, only the ARES cavities were used. For details regarding the rf systems of KEKB, see Refs. [3,4]. The rf issues to be considered for the heavy-beam current storage are summarized in Ref. [10]. 
TABLE I. rf-related design parameters of KEKB.

\begin{tabular}{lcc}
\hline \hline Parameter & LER & HER \\
\hline Beam energy [GeV] & 3.5 & 8.0 \\
Beam current [A] & 2.6 & 1.1 \\
Bunch gap length [\%] & 5 & 5 \\
Beam power [MW] & 4.5 & 4.0 \\
Bunch length [mm] (rms) & 4 & 4 \\
rf frequency [MHz] & \multicolumn{2}{c}{508.887} \\
Harmonic number & \multicolumn{2}{c}{5120} \\
Revolution frequency [kHz] & ARES & 99.4 \\
Cavity type & 20 & SCC/ARES \\
Number of cavities & 10 & $8 / 12$ \\
Total rf voltage $[\mathrm{MV}]$ & 14.8 & 18 \\
$R / Q$ of cavity $[\Omega]$ & 3.0 & $93 / 14.8$ \\
Loaded Q of cavity [×10 & $7.0 / 3.0$ \\
Coupling factor $\left(\beta_{\pi / 2}\right)$ & 2.7 & $-/ 2.7$ \\
rf voltage/cavity [MV] & 0.5 & $1.5 / 0.5$ \\
Wall loss/cavity $[\mathrm{kW}]$ & 154 & $-/ 154$ \\
Beam power/cavity $[\mathrm{kW}]$ & 221 & $240 / 173$ \\
Number of klystrons & 10 & $8 / 6$ \\
Klystron power $[\mathrm{kW}]$ & $\sim 810$ & $\sim 270 / \sim 730$ \\
\hline \hline
\end{tabular}

KEKB was operated with a gap of the length of $5 \%$ to $10 \%$ of the ring. The bunch phase shift along the train was measured and compared with a time-domain simulation [11-14]. In most of the train, except for the leading part, the measured phase shift agreed well with the simulation result and with an estimate obtained from a simple analytical form. However, a rapid phase change was observed at the leading part of the train, which was not predicted by the simulation or by the analytical form. The cause of the rapid change was suspected to be excitation of parasitic 0 and $\pi$ modes of ARES due to transient beam-loading. Thus, we

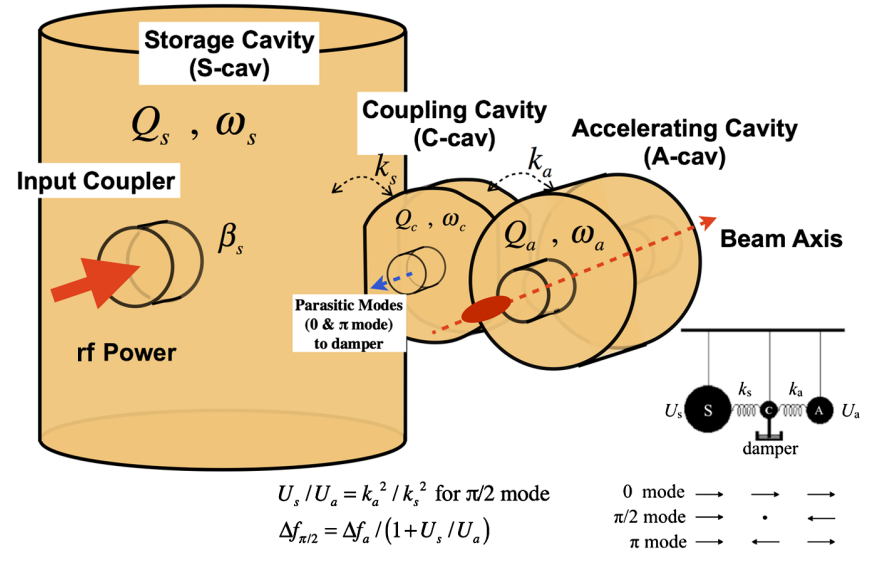

FIG. 1. Illustration of the ARES cavity structure (Ref. [9]). The accelerating (A-)cavity is coupled to the storage (S-)cavity via a coupling (C-)cavity. The $\mathrm{C}$-cavity has a parasitic-mode (0 and $\pi$-mode) damper. An illustration of the coupled pendulum model is also shown. developed an advanced simulation that treats transient loading in each of the cavities of the three-cavity system of ARES instead of the equivalent single cavity, operating in the accelerating mode, used in the previous simulation. In this paper, we will show that the new simulation clearly reproduces the rapid phase change in the leading part of the train, which now agrees well with the measurement. Thus, we will clarify that the rapid phase change is caused by excitation of parasitic 0 and $\pi$ modes of ARES due to the gap transient.

KEKB is being upgraded to SuperKEKB, which is aiming at a 40 times higher luminosity than KEKB $[15,16]$. The rf systems are being reinforced to handle twice as large stored beam currents in both rings and much higher beam power (compared to KEKB) [17]. ARES and SCC will be reused with the improvements required for the input couplers and HOM dampers. The rf power source systems, including klystrons, waveguides, and cooling systems, also need to be reinforced to increase the driving rf power to provide larger beam power. Furthermore, a new low-level rf (LLRF) control system, which is based on a recent digital control technique using field-programmable gate arrays (FPGAs), has been developed to realize higher accuracy and greater flexibility [18]. For nine rf stations, among a total of thirty, the LLRF control system used in KEKB $[19,20]$ has been replaced with new ones. The gap transient for SuperKEKB was studied using the new simulation, and the rapid phase change was found to be much larger than for KEKB due to higher beam currents. The main issue due to the gap transient is the difference in beam phase change along the bunch train between the two rings, which is caused by the difference in the beam current and the rf cavity configurations. This paper will also present the results of gap transients in SuperKEKB and suggest measures to avoid possible beam deterioration arising from beam-beam effects at the collision point by means of mitigation of the relative beam phase difference between the two rings.

The paper is organized as follows. In the next section, we describe the gap transient effect observed in KEKB. Then, in Sec. III, we develop our simulation method for the coupled cavities of ARES, which then is compared to data in Sec. IV. Section V predicts what to expect for SuperKEKB and Sec. VI suggests a couple of ways to mitigate potential issues. We conclude our paper in Sec. VII.

\section{BUNCH GAP TRANSIENT EFFECT IN KEKB}

Assuming that the cavity is operated at optimum tuning and that the revolution period $T_{R}$ is much shorter than the filling time of the cavity $T_{f}: T_{R} / T_{f} \ll 1$, the cavity phase shift $\Delta \phi$ caused by a bunch gap transient can be estimated analytically by [21] 


$$
\Delta \phi=\frac{\omega_{r f} I_{b}}{2 V_{\mathrm{acc}}}\left(\frac{R}{Q}\right) \Delta t_{g}=\frac{P_{b} \Delta t_{g}}{2 \cos \phi_{s} U}
$$

where $\omega_{r f}$ is the angular rf frequency, $I_{b}$ the stored beam current, $V_{\text {acc }}$ the accelerating voltage, $R$ the shunt impedance of the cavity, $Q$ its Q-value, $\Delta t_{g}$ the transient time of the gap, $P_{b}$ the beam power provided by the cavity, $\phi_{s}$ the synchronous phase, and $U$ the energy stored in the cavity. Equation (1) indicates that a larger stored energy and shorter gap reduce the phase shift. In the case of KEKB operation with a 2.6-A beam current and 5\% bunch gap, an Eq. (1) estimate gives that $\Delta \phi$ is about 3 degrees. The amplitude modulation $\Delta V / V$ should also be counted to evaluate the effective phase change $\Delta \phi_{\text {eff }}$ in the beam phase as

$$
\Delta \phi_{\mathrm{eff}}=\Delta \phi+\frac{\Delta V}{V} \frac{1}{\tan \phi_{s}}
$$

For the case of ARES in KEKB operation, $T_{R} / T_{f}=0.54$, which is not enough to satisfy the approximation condition $T_{R} / T_{f} \ll 1$ for Eq. (1). Thus, strict evaluations using a time-domain simulation of the bunch gap transient effect are required. The time-domain simulation results for KEKB operation were reported in Refs. [20,22]. The simulations employed in these reports used equivalent single-cell cavities, operating in the accelerating mode, for the ARES cavity system. The relative phase difference between the LER and HER at the collision point was estimated to be within 0.5 degrees $\left(=0.2 \sigma_{z}\right)$, where $\sigma_{z}$ is the bunch length. This is regarded acceptable in the design of KEKB [23].

The measurement of the beam phase was performed during a collision using a gated bunch-by-bunch beam monitoring system in the KEKB operation. The observed results were published in Refs. [11-14]. Figure 2 shows examples of the observations together with the simulation results $[11,12]$. The abscissa axis in the figure is the bucket number, and the ordinate is the measured beam phase. In this case, the empty gap is $10 \%$. The phase error of the gate module is less than 0.3 degrees in these measurements [12]. The measured phase shift along the bunch train agrees well with the simulation in most parts of the train. However, a rapid phase change of a few degrees is found at the leading part of the train up to a bucket number of about 400 , which was not predicted by the simulation. The cause of the rapid phase change in the leading bunches was suspected to be attributed to the parasitic modes of the ARES cavity because the phase change duration is consistent with the filling time (or the Q-value) of the C-cavity of ARES [13]. In order to establish this supposition, we advanced the simulation approach by accounting for beam loading in the three-cavity system of ARES. This will be presented in the next section.

\section{SIMULATION METHOD OF THE ARES BEHAVIOR WITH BEAM LOADING}

To analyze the bunch gap transient effect in KEKB and SuperKEKB, a new simulation code was developed by extending the time-evolutionary calculation of a single cavity to the three-cavity system of the ARES structure. Details of the new simulation method are described below.

\section{A. Resonator expression for time-evolutionary simulation for a single-cell cavity}

As a first step, a single cell cavity is considered for the simulation. Time evolutionary behavior of the baseband signal (or envelop) of a resonator with angular resonant frequency $\omega_{0}$, which is excited at the angular frequency $\omega_{r f}$, can be calculated approximately by the first order differential equations of time $t$ given by [24]:
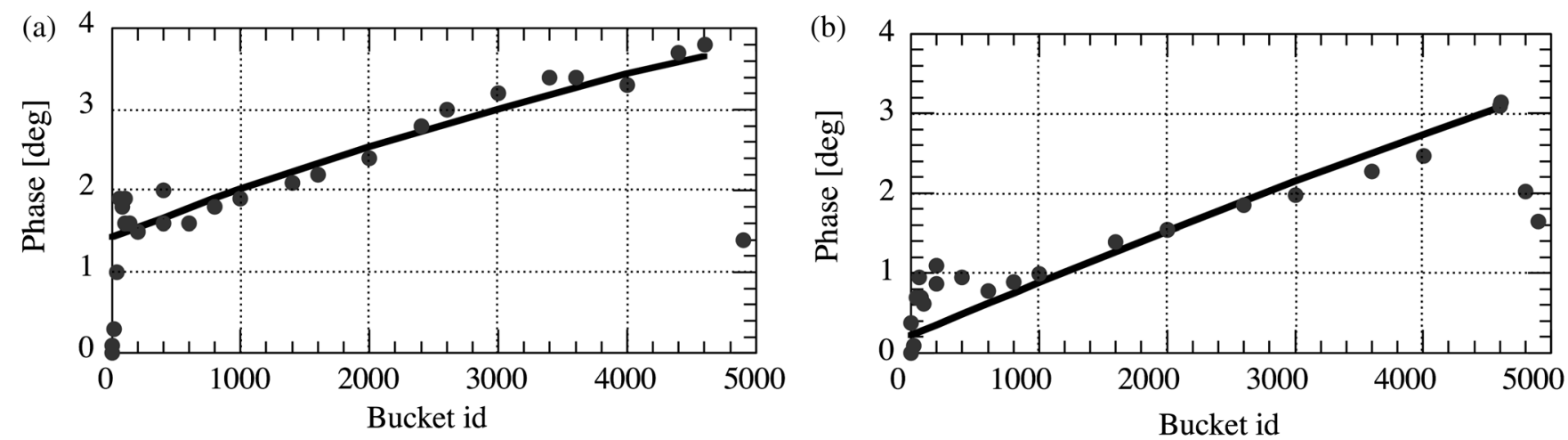

FIG. 2. Examples of the observed phase modulation caused by a bunch gap transient in KEKB operation. (a) LER with a beam current of $660 \mathrm{~mA}$ and (b) HER with $470 \mathrm{~mA}$. The solid lines indicate the results of the simulation reported in Ref. [11], which used equivalent single-cell cavities operating in the accelerating mode. 


$$
\begin{aligned}
& \left\{\begin{array}{l}
\dot{V}_{r}(t)+\omega_{1 / 2}(1+\beta) V_{r}(t)+\Delta \omega V_{j}(t)=R \omega_{1 / 2} I_{g r}(t) \\
\dot{V}_{j}(t)+\omega_{1 / 2}(1+\beta) V_{j}(t)-\Delta \omega V_{r}(t)=R \omega_{1 / 2} I_{g j}(t)
\end{array},\right. \\
& \omega_{1 / 2}=\frac{\omega_{0}}{2 Q_{0}}, \quad \Delta \omega=\omega_{0}-\omega_{r f}
\end{aligned}
$$

where $Q_{0}$ and $\beta$ are the unloaded Q-value and input coupling factor, respectively, and $V$ and $I_{g}$ are the baseband components of the cavity voltage and driving current, respectively. The suffices $r$ and $j$ indicate the real and imaginary parts (or I/Q components) of the vector quantities, respectively. In the following description, a boldfaced variable represent the vector variable coupling the real and imaginary parts as $\mathbf{X}=\left(X_{r}, X_{j}\right)$.

In Eq. (3), it is inconvenient to assign actual values to $\mathbf{I}_{g}$ and $R$. Thus, to compute the cavity voltage $\mathbf{V}$, a voltageequivalent variable is preferred over a current variable for

the driving term. Therefore, in our calculation, the driving term $R \mathbf{I}_{g}$ is replaced with the voltage-equivalent variable $\mathbf{V}_{g}$ :

$$
R \mathbf{I}_{g}=2 \sqrt{\beta} \mathbf{V}_{g} .
$$

Here, the driving quantity $\mathbf{V}_{g}$ is treated as of the same dimension as $\mathbf{V}$. The reflection voltage $\mathbf{V}_{R}$ is also given by

$$
\mathbf{V}_{R}=\mathbf{V} \sqrt{\beta}-\mathbf{V}_{g} .
$$

By rewriting Eq. (3) using Eq. (4), transforming it into a computable discrete form, and adding a beam-induced term, a recurrence formula for the time-evolutionary calculation, including beam loading, is obtained as

$$
\begin{aligned}
{\left[\begin{array}{l}
V_{r}^{n+1} \\
V_{j}^{n+1}
\end{array}\right]=} & {\left[\begin{array}{cc}
1-(1+\beta) \Delta t \cdot \omega_{1 / 2} & -\Delta t \Delta \omega \\
\Delta t \Delta \omega & 1-(1+\beta) \Delta t \cdot \omega_{1 / 2}
\end{array}\right]\left[\begin{array}{c}
V_{r}^{n} \\
V_{j}^{n}
\end{array}\right] } \\
& +2 \sqrt{\beta} \Delta t \cdot \omega_{1 / 2}\left[\begin{array}{c}
V_{g r}^{n} \\
V_{g j}^{n}
\end{array}\right]+(1+\beta) \Delta t \cdot \omega_{1 / 2}\left[\begin{array}{c}
V_{b r}^{n} \\
V_{b j}^{n}
\end{array}\right],
\end{aligned}
$$

where the superscript $n$ denotes the index of the time step discretized with a time interval of $\Delta t(t=n \Delta t)$ and $\mathbf{V}_{b}$ is the beam-induced voltage. Equation (6) gives a good approximation of the cavity response under the condition that $\Delta t$ is small enough compared to the cavity filling time $\left(\Delta t \ll Q_{L} / \omega_{0}\right)$ for the loaded Q-value $Q_{L}\left(=Q_{0} /(1+\beta)\right)$ and small detuning of $\Delta \omega / \omega_{0} \ll 1$. The transient effect of the bunch gap is incorporated in $\mathbf{V}_{b}$ by setting $\mathbf{V}_{b}$ to zero in the empty gap. Because the dimension of the beam loading is defined the same as that of the cavity voltage in Eq. (6), the amplitude of $\mathbf{V}_{b}$ for the simulation is given by

$$
\left|\mathbf{V}_{b}\right|=I_{b} \cdot Q_{L}\left(\frac{R}{Q}\right)
$$

In the simulation, the average value of $\mathbf{V}$ is kept constant by controlling the driving voltage $\mathbf{V}_{g}$ with a regulation-loop function for the cavity voltage. The details of the regulation loop are described in Sec. III C. Equations (6) and (7) are directly adaptable to SCCs, which are single cell cavities.

\section{B. Extension to the three-cavity system of the ARES}

To include the three-cavity system of ARES in the timeevolutional simulation, we extend Eq. (6) to a threeresonator coupling formula by adding coupling terms for the accelerating mode pass band comprising the $0, \pi / 2$, and $\pi$ modes, which gives

$$
\left[\begin{array}{l}
V_{a r}^{n+1} \\
V_{a j}^{n+1} \\
V_{c r}^{n+1} \\
V_{c j}^{n+1} \\
V_{s r}^{n+1} \\
V_{s j}^{n+1}
\end{array}\right]=\left[\begin{array}{cccccc}
W_{a} & -D_{a} & 0 & K_{a a} & 0 & 0 \\
D_{a} & W_{a} & -K_{a a} & 0 & 0 & 0 \\
0 & K_{a c} & W_{c} & -D_{c} & 0 & K_{s c} \\
-K_{a c} & 0 & D_{c} & W_{c} & -K_{s c} & 0 \\
0 & 0 & 0 & K_{s s} & W_{s} & -D_{s} \\
0 & 0 & -K_{s s} & 0 & D_{s} & W_{s}
\end{array}\right]\left[\begin{array}{c}
V_{a r}^{n} \\
V_{a j}^{n} \\
V_{c r}^{n} \\
V_{c j}^{n} \\
V_{s r}^{n} \\
V_{s j}^{n}
\end{array}\right]+\left[\begin{array}{c}
-B \cdot V_{b r}^{n} \\
-B \cdot V_{b j}^{n} \\
0 \\
0 \\
G \cdot V_{g r}^{n} \\
G \cdot V_{g j}^{n}
\end{array}\right] .
$$


The coefficients in Eq. (8) are defined in terms of the cavity parameters as

$$
\left\{\begin{array}{l}
\Delta \omega_{\mu}=\omega_{\mu}-\omega_{r f}, \quad \omega_{\mu / 2}=\frac{\omega_{\mu}}{2 Q_{\mu}}, \\
G=2 \sqrt{\beta_{s}} \Delta t \cdot \omega_{s / 2}, \quad C=\sqrt{\beta_{s}}, \quad B=\Delta t \cdot \omega_{a / 2}, \\
W_{a}=1-\Delta t \cdot \omega_{a / 2}, \quad W_{c}=1-\Delta t \cdot \omega_{c / 2}, \\
W_{s}=1-\left(\beta_{s}+1\right) \cdot \Delta t \cdot \omega_{s / 2}, \quad D_{\mu}=\Delta t \cdot \Delta \omega_{\mu}, \\
K_{\mu \nu}=\frac{k_{\mu}}{2} \cdot \Delta t \cdot \omega_{\nu / 2} \cdot Q_{\nu}=\frac{k_{\mu} \cdot \Delta t \cdot \omega_{\nu}}{4}, \\
\mu, \nu: a, \mathrm{c}, \mathrm{s},
\end{array}\right.
$$

where the parameters with suffices a, c, and s denote those of the A-, C- and S-cavities, respectively, and the suffices $\mu$ and $\nu$ stand for any one of the suffices a, c, and s. Here, $\omega_{\mu}$ is the resonant frequency of each cavity (see Fig. 1). The parameters $k_{a}$ and $k_{s}$ are the coupling factors between the A- and C-cavities and the $\mathrm{S}$ - and C-cavities, respectively.

The Q-value of the S-cavity $Q_{s}$ is defined as the unloaded Q-value in Eqs. (8) and (9). We define the input-coupling factor $\beta_{s}$ for the S-cavity as $\beta_{s}=Q_{s} / Q_{\text {ext }}$, where $Q_{\text {ext }}$ is the external Q-value for the input coupler implemented at the S-cavity. The coupling factor for the complete three-cavity system, $\beta_{\pi / 2}$, is reduced by the ratio $Q_{\text {tot }} / Q_{s}$, where $Q_{\text {tot }}$ is the total Q-value of the three-cavity system [5]. Accordingly, in the simulation, $\beta_{s}$ is set to $\beta_{\pi / 2} \times Q_{s} / Q_{\mathrm{tot}}$. The ratio $Q_{\mathrm{tot}} / Q_{s}$ is approximately 0.7 for ARES.

In Eq. (8), the driving voltage $\mathbf{V}_{g}$ excites the S-cavity voltage $\mathbf{V}_{s}$ via the coupling factor $\beta_{s}$ and then the reflection voltage from the S-cavity is obtained from Eq. (5) with $\beta=\beta_{s}$. The beam-induced voltage $\mathbf{V}_{b}$ excites the A-cavity voltage $\mathbf{V}_{a}$. For the case of ARES, $\mathbf{V}_{b}$ is given by

$$
\left|\mathbf{V}_{b}\right|=I_{b} \cdot Q_{a}\left(\frac{R}{Q}\right)_{a}=I_{b} \cdot Q_{a}\left(\frac{R}{Q}\right)\left(\frac{U_{\mathrm{tot}}}{U_{a}}\right),
$$

where $(R / Q)_{a}$ is the $R / Q$ parameter for the A-cavity, $U_{\text {tot }}$ is the total stored energy of the three cavities ( $U_{\text {tot }}=U_{s}+U_{c}+U_{a}$ ) and $U_{\mu}$ is the stored energy of the corresponding cavity. For the $\pi / 2$ mode of ARES, because $U_{c}$ is negligibly small compared to $U_{s}$ and $U_{a}, U_{\text {tot }} / U_{a} \approx 1+U_{s} / U_{a} \approx 1+\left(k_{a} / k_{s}\right)^{2} \approx 10$.

In ARES, the cavity detuning of the $\pi / 2$ mode, $\Delta \omega_{\pi / 2}$, is related to the A-cavity detuning $\Delta \omega_{a}$ as $[6,25]$,

$$
\Delta \omega_{\pi / 2}=\Delta \omega_{a} \times\left(\frac{U_{a}}{U_{\text {tot }}}\right) \approx \frac{\Delta \omega_{a}}{1+\left(k_{a} / k_{s}\right)^{2}} .
$$

Therefore, the frequency shift for the optimum tuning, compensating for the reactive component of the beam loading, is reduced to $1 / 10$ of the A-cavity detuning $\Delta \omega_{a}$.

\section{Cavity voltage regulation and tuning control}

In the usual operation of storage rings, the cavity voltage has to be kept constant under beam loading by increasing the rf driving power with the cavity detuning. The amplitude and phase of the cavity voltage are regulated by an LLRF control system, and the cavity tuner is controlled to maintain the cavity transmission phase. For the KEKB operation, the amplitude feedback loop and phase lock loop are applied individually to the A-cavity voltage regulation of ARES [19,20]. In the new LLRF control system, consisting of several FPGAs for the SuperKEKB operation, I/Q components will be handled digitally by using an I/Q modulator.

However, according to the response characteristics of a closed loop for cavity-field regulation, the feedback control contributes little to the compensation of the bunch gap transient, and the phase change depends mostly on the cavity condition [26]. Feedforward control is also unavailable to cancel this effect, because the klystron is incapable to output enough power to compensate for the transient effect, and the bandwidth of the klystron is only $100 \mathrm{kHz}$, while the required bandwidth for the compensation is greater than $500 \mathrm{kHz}$ [22].

During the ARES cavity operation, the tuning of both the $\mathrm{S}$ - and A-cavities is controlled by the LLRF control system. The S-cavity tuner is controlled to adjust the phase between the input rf and the S-cavity pick-up monitor. The A-cavity is tuned by adjusting the phase between the C-and A-cavity pick-up monitor signals. The optimum detuning for canceling the reactive component of the beam loading is automatically realized by the tuning-phase adjustment. The cavity tuner is a mechanical plunger moved by a pulse motor mounted at each cavity, thus the tuner control response is much slower than the amplitude and phase control. For the case of SCC tuning, a piezo actuator is combined with a mechanical tuner for fine-tuning in a narrow bandwidth (high Q-value). The tuner control response for SCC is also much slower than the gap transient effect.

In our simulation, we implement the cavity-voltage regulation loop and the A-cavity tuning control for realistic modeling of the operation, as shown in Fig. 3.

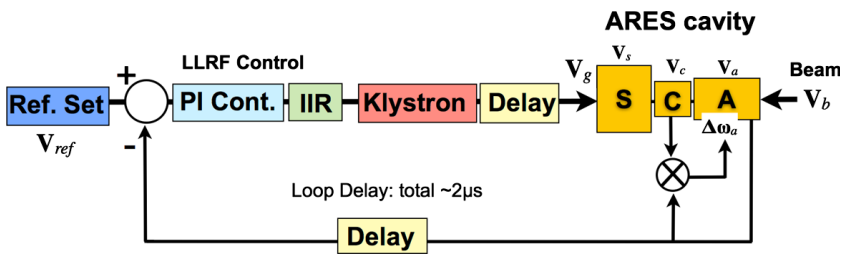

FIG. 3. Implementation of feedback loops in the simulation. 
A proportional and integral (PI) feedback control with an infinite impulse response (IIR) filter is applied to the I/Q components (the real and imaginary parts) of the cavity voltage in the simulation in the same way as the new LLRF control system for SuperKEKB. The PI control acts on each component of the I/Q individually. The feedback gains of the PI control and the loop delay are assigned with the practical condition parameters reported in Ref. [26] and a first-order lag filter is applied for the IIR and the klystronfrequency property.

By applying the regulation loop function to the cavity voltage, the average value of $\mathbf{V}_{a}$ along the train with beam loading is kept constant. For the simulation of ARES tuning/detuning control, the A-cavity detuning parameter $\Delta \omega_{a}$ is varied to cancel the phase change between the A-cavity $\left(\mathbf{V}_{a}\right)$ and the C-cavity voltage $\left(\mathbf{V}_{c}\right)$ under the beam loading in the simulation. The tuner control response is set much slower than the voltage regulation and the revolution period, just as in the actual operation. Therefore, $\Delta \omega_{a}$ is slowly incremented or decremented by a constant value to simulate the step moving of the pulse motors. In our simulation, the (de-)tuning control is applied to the A-cavity only, as shown in Fig. 3, because the reactive component of the beam loading is compensated only by the A-cavity detuning, keeping $\Delta \omega_{s}=0$. For the SCC simulation, the same above-mentioned function is implemented to calculate Eq. (6).

\section{SIMULATION OF BUNCH GAP TRANSIENT IN KEKB WITH NEW APPROACH}

The phase modulation $\Delta \phi$ of the cavity voltage due to the bunch gap transient effect was evaluated by using the new simulation method. First, the KEKB operation was simulated to understand the measurements shown in Fig. 2, reported in Ref. [11]. Table II shows the operation
TABLE II. KEKB operation parameters used for the simulation of the observed condition.

\begin{tabular}{lcc}
\hline \hline & $\begin{array}{c}\text { LER } \\
\text { (ARES) }\end{array}$ & $\begin{array}{c}\text { HER } \\
\text { (SCC/ARES) }\end{array}$ \\
\hline Beam current [A] & 0.66 & 0.47 \\
Bunch gap length [\%] & 10 & 10 \\
Beam power/cavity [kW] & 68 & $165 / 72$ \\
rf voltage/cavity [MV] & 0.33 & $1.25 / 0.35$ \\
Number of cavity & 16 & $6 / 10$ \\
$R / Q$ of cavity $[\Omega]$ & 14.8 & $93 / 14.8$ \\
Loaded Q of cavity $\left[\times 10^{4}\right]$ & 3.0 & $7.0 / 3.0$ \\
Coupling factor & 2.7 & $-/ 2.7$ \\
Wall loss/cavity $[\mathrm{kW}]$ & 100 & $-/ 105$ \\
Acc. phase $\phi$ acc $[\mathrm{deg}]$ & 72 & $74 / 64(*)$ \\
Cavity detuning $\Delta f[\mathrm{kHz}]$ & $-8.1(-81)$ & $-9.5 /-5.1(-51)$ \\
$\quad$ (A-Cav detuning of ARES) & \multicolumn{2}{c}{} \\
\hline \hline
\end{tabular}

(*) Synchronous phase of HER: 71 degrees.

TABLE III. ARES cavity parameters used for the simulation.

\begin{tabular}{lc}
\hline \hline Q-value of A-cavity $\left(Q_{a}\right)$ & 26000 \\
Q-value of C-cavity $\left(Q_{c}\right)$ & 100 \\
Q-value of S-cavity $\left(Q_{s}\right)$ & 180000 \\
Coupling between A and C cavity $\left(k_{a}\right)$ & $5 \%$ \\
Coupling between S and C cavity $\left(k_{s}\right)$ & $1.6 \%$ \\
\hline \hline
\end{tabular}

conditions related to the cavities when the data were obtained. These parameters are used in the simulation, including the bunch gap of $10 \%$ applied to $\mathbf{V}_{b}$ in Eq. (8) for ARES and in Eq. (6) for SCC. In addition, the parameters for the three-cavity system of ARES used for the simulation are shown in Table III.

The simulation results are shown in Figs. 4(a) and 4(b) for LER and HER, respectively. The plotted phase is the
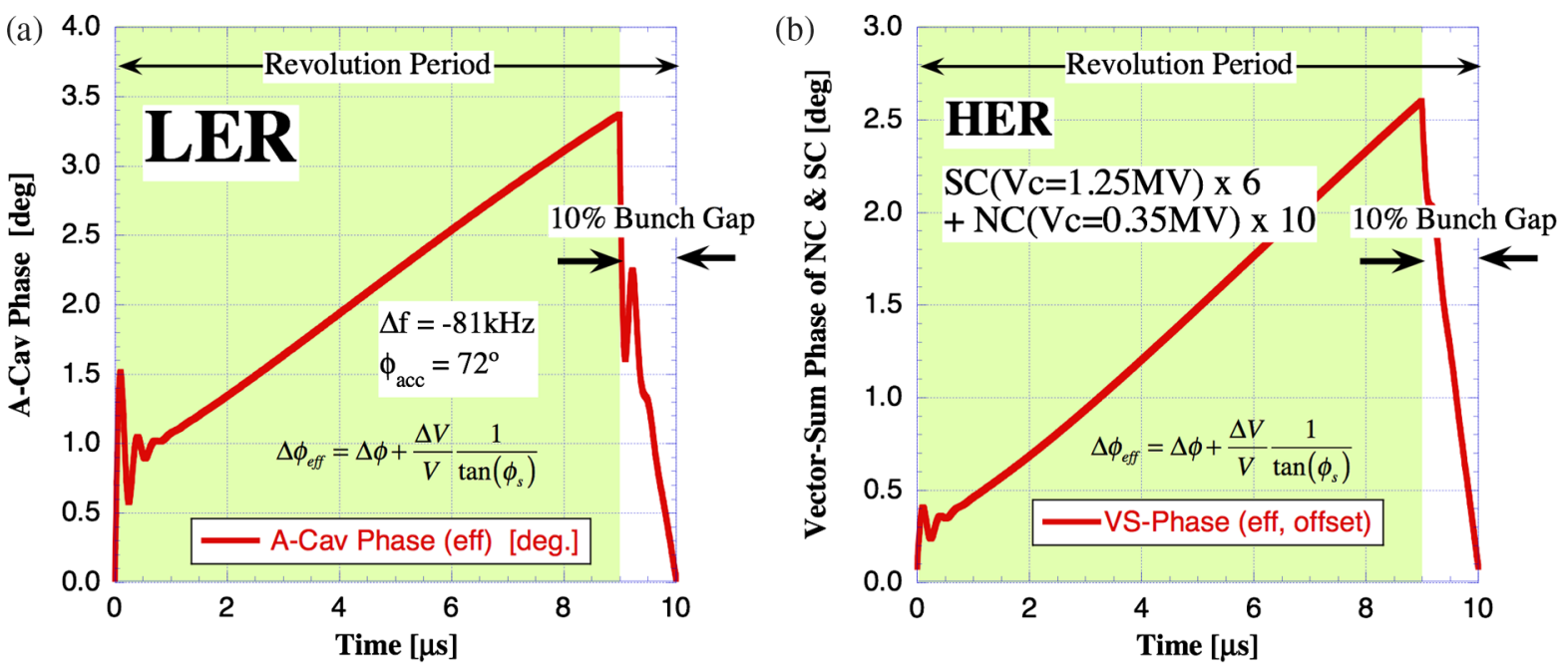

FIG. 4. Simulation results of the phase modulation due to a $10 \%$ bunch gap for KEKB operation. (a) LER with the ARES cavities only and (b) the vector sum of ARES and SCC for HER. 

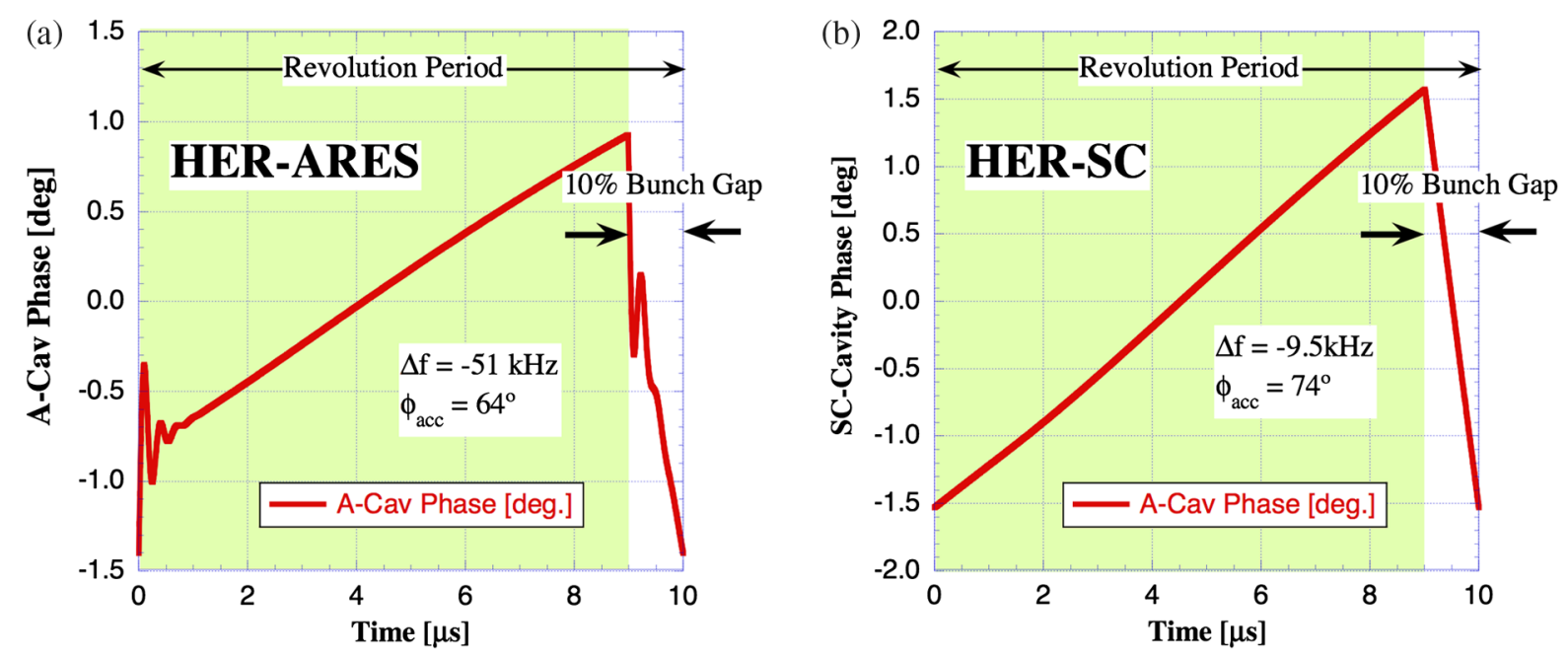

FIG. 5. Simulation results of the phase modulation in the ARES cavities (a) and SCCs (b) for HER to calculate the vector sum shown in Fig. 4(b).

effective phase change $\Delta \phi_{\text {eff }}$ given by Eq. (2), where the amplitude modulation is taken into account. For LER operating with only ARES, the same operation condition is adopted for all ARES cavities. On the other hand, for HER, the vector sum voltage of the two types of cavities, eight ARES cavities and six SCCs, is calculated. The simulation results of the ARES and the SCC phase modulation for HER, which are the basis for the calculation of the vector sum voltage, are shown in Figs. 5(a) and 5(b), respectively. In these simulations, the revolution frequency is set to $100 \mathrm{kHz}$ instead of $99.4 \mathrm{kHz}$ to simplify the abscissa axis of time, and the time step of the calculation $\Delta t$ is $10 \mathrm{~ns}$. Time 0 corresponds to the head bunch of the bunch train, and the $10 \%$ empty gap is located between 9 to $10 \mu \mathrm{s}$.

To perform a more strict comparison with the observations, the LER simulation was done again using the actual revolution frequency of $99.4 \mathrm{kHz}$. Figure 6 shows the simulation result (dashed red line) into the bucket-id coordinate. In this figure, the observed data of Fig. 2 are

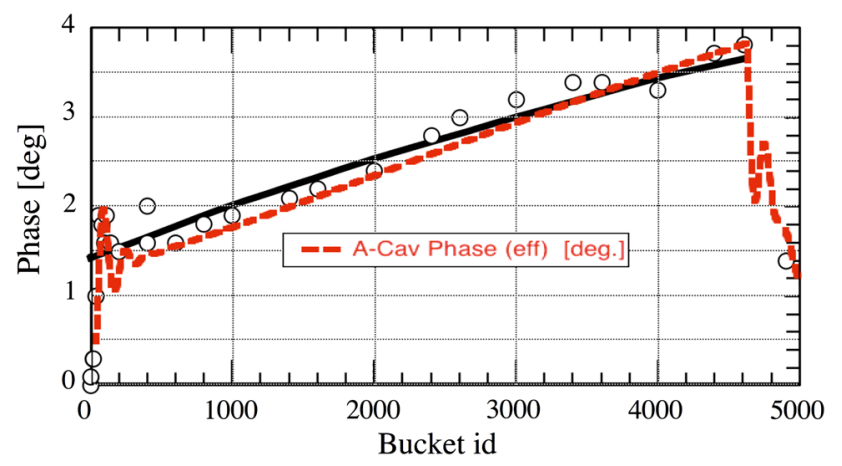

FIG. 6. Comparison of the result of the new simulation for LER (dashed red line) with the observed data (dots) and the previous simulation (solid black line). The observed data and the previous simulation are replotted from Fig. 2(a). replotted for comparison. The overall features of the phase modulation in the simulation results agree well with the observation within the measurement error of 0.3 degrees. In particular, the observed rapid phase change and the ringing at the leading part of the bunch train, which was not predicted by the previous simulation, is reproduced by the new simulation for the ARES cavity, while such a rapid phase change does not appear in the calculation with only SCC as shown in Fig. 5(b). From these simulation results, we conclude that the rapid phase change in the aftermath of the gap is reproduced by including the three-cavity system

TABLE IV. SuperKEKB parameters for the estimation of the bunch gap transient effect.

\begin{tabular}{lcc}
\hline \hline Parameter & LER & HER \\
\hline Beam energy [GeV] & 4 & 7 \\
Beam current [A] & 3.6 & 2.6 \\
Bunch gap length [\%] & 2 & 2 \\
Beam power [MW] & 8 & 8.3 \\
Bunch length [mm] (rms) & 6 & 5 \\
rf frequency [MHz] & \multicolumn{2}{c}{508.887} \\
Harmonic number & \multicolumn{2}{c}{5120} \\
Revolution frequency [kHz] & ARES & SCC/ARES \\
Cavity type & 22 & $8 / 8$ \\
Number of cavities & $10-11$ & $15-16$ \\
Total rf voltage [MV] & 2.4 & $7.0 / 2.0$ \\
Loaded Q of cavity [×10 ${ }^{4}$ ] & 4.3 & $-/ 5$ \\
Coupling factor $(\beta)$ & 0.48 & $1.5 / 0.5$ \\
rf voltage/cavity [MV] & 140 & $-/ 150$ \\
Wall loss/cavity [kW] & 460 & $400 / 600$ \\
Beam power/cavity [kW] & $-28(-280)$ & $-18 /-44(-180)$ \\
Cavity detuning [kHz] & \multicolumn{2}{c}{$8 / 8$} \\
$\quad$ A-Cav detuning of ARES) & 18 & $\sim 450 / \sim 800$ \\
Number of klystrons & $\sim 600$ & \multicolumn{2}{c}{ Klystron power [kW] } \\
\hline \hline
\end{tabular}



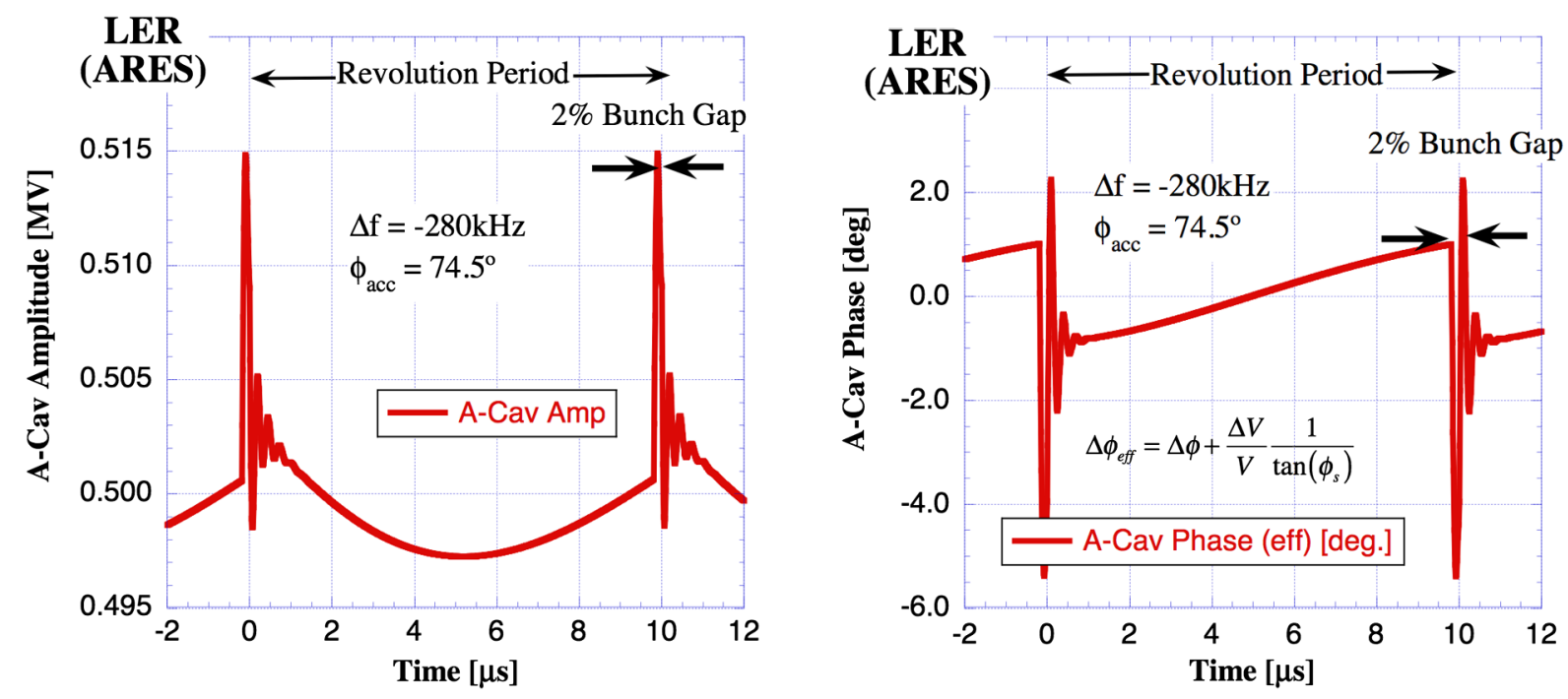

FIG. 7. Simulation results of the amplitude (left) and phase (right) modulation due to the $2 \%$ bunch gap in ARES. The calculations are done for LER at SuperKEKB.

of ARES. The ringing duration definitely depends on the Q-value of the C-cavity $\left(Q_{c}\right)$, and it is consistent with the filling time of the C-cavity. Consequently, it is concluded that the rapid phase change in the leading part of the train is attributed to parasitic mode ( 0 and $\pi$ mode) excitations, or in other words, the mode dispersion in the ARES cavity. The ringing of the phase corresponds to a beat with the 0 or $\pi$ mode, the frequency of which is separated by about $3 \mathrm{MHz}$ from the $\pi / 2$ mode [4]; the beat period is consistent with the $3-\mathrm{MHz}$ mode separation. Thus, the apparent cause of the rapid phase change is demonstrated by our new simulation.

In Fig. 6, there still appear slight differences between the measurement and the simulation, which may be caused by some other effects. One of the possible other effects in the data that are not captured in the model is beam phase oscillation due to longitudinal coupled-bunch instability associated with the detuning of the cavities (so called $\mu=-1$ mode). Actually, the oscillation caused by this instability was often observed during the KEKB operation. Another point is that in the measurement one data point at the bucket ID of about 400 significantly deviates from other data points. The reason of the outlier of the data at bucket ID of about 400 is not clear. In another measurement this outlier was not observed (see, for example, Ref. [12]). We cannot identify if this outlier is caused by physical phenomenon or not.

\section{EVALUATION OF BUNCH GAP TRANSIENT EFFECT FOR SUPERKEKB}

Next, the phase modulation caused by the bunch gap transient was estimated for SuperKEKB by using the new simulation method. The operation parameters used for the simulation are shown in Table IV. In SuperKEKB, the stored beam currents will be approximately twice as high as those in KEKB operation were (LER $2 \mathrm{~A}$ and HER 1.4 A). The beam power per cavity will be more than twice as high as those of KEKB were. Therefore, the input-coupling factor of the ARES cavity should be increased and a larger cavity detuning will be required for the beam loading compensation. On the other hand, the length of the bunch gap will be reduced from $5 \%$ of the ring in KEKB to $2 \%$ of the ring in SuperKEKB by improving the rise time of the abort kicker.

Figure 7 shows the simulation result of the amplitude and phase modulation in the ARES cavity caused by the bunch

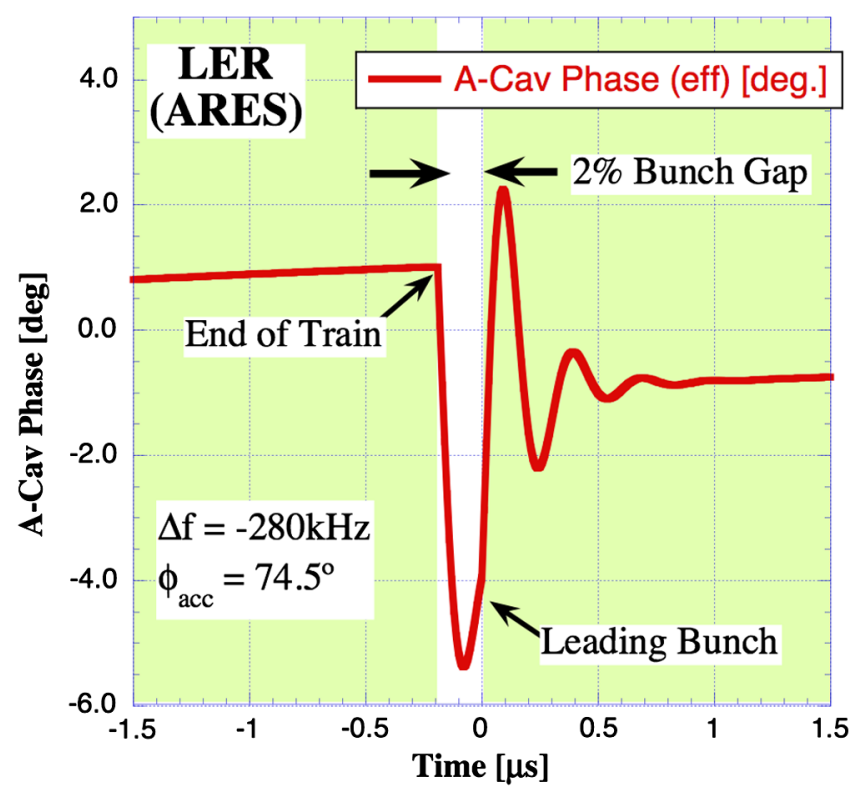

FIG. 8. A plot of the simulated phase modulation shown in Fig. 7, enlarged to show the empty gap region. 

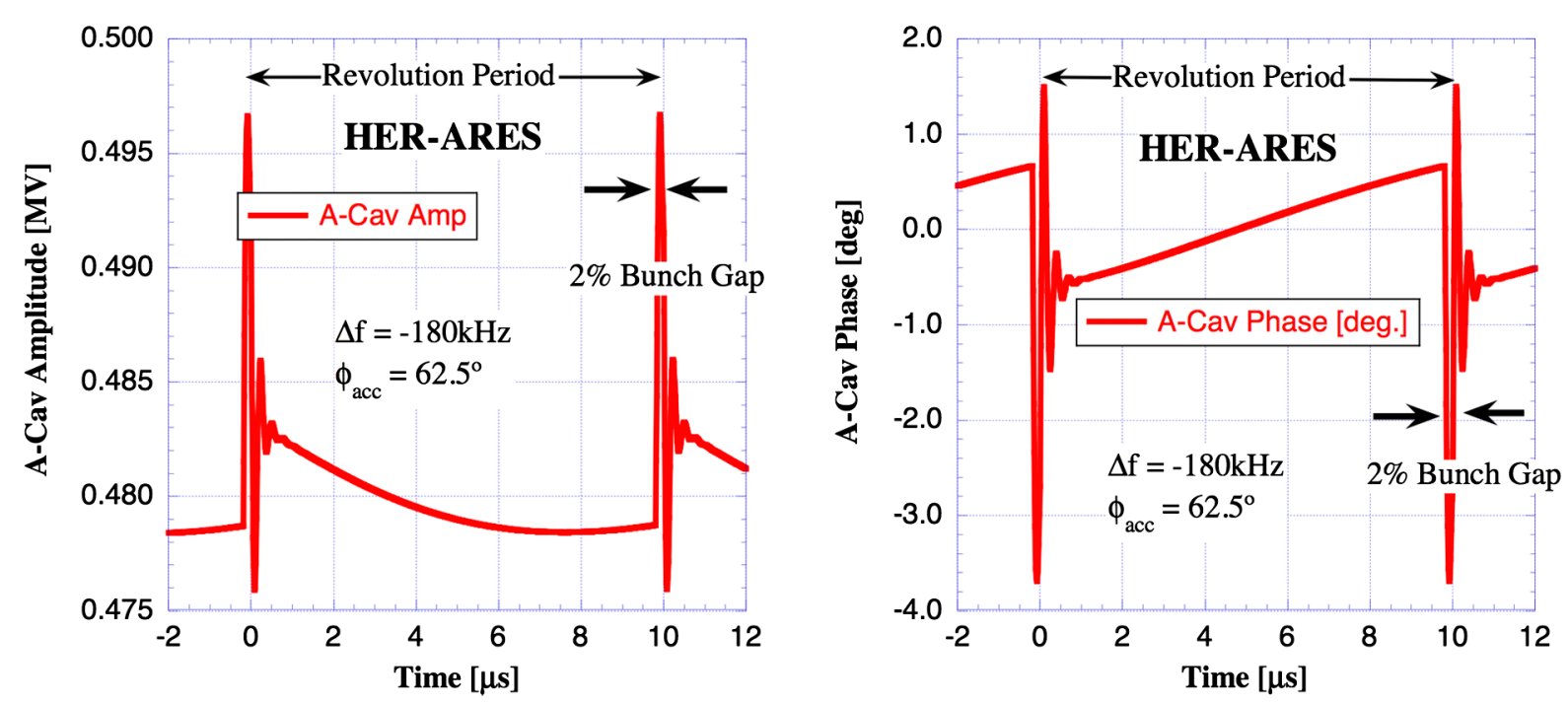

FIG. 9. ARES voltage (amplitude and phase) modulation simulated for HER in SuperKEKB.
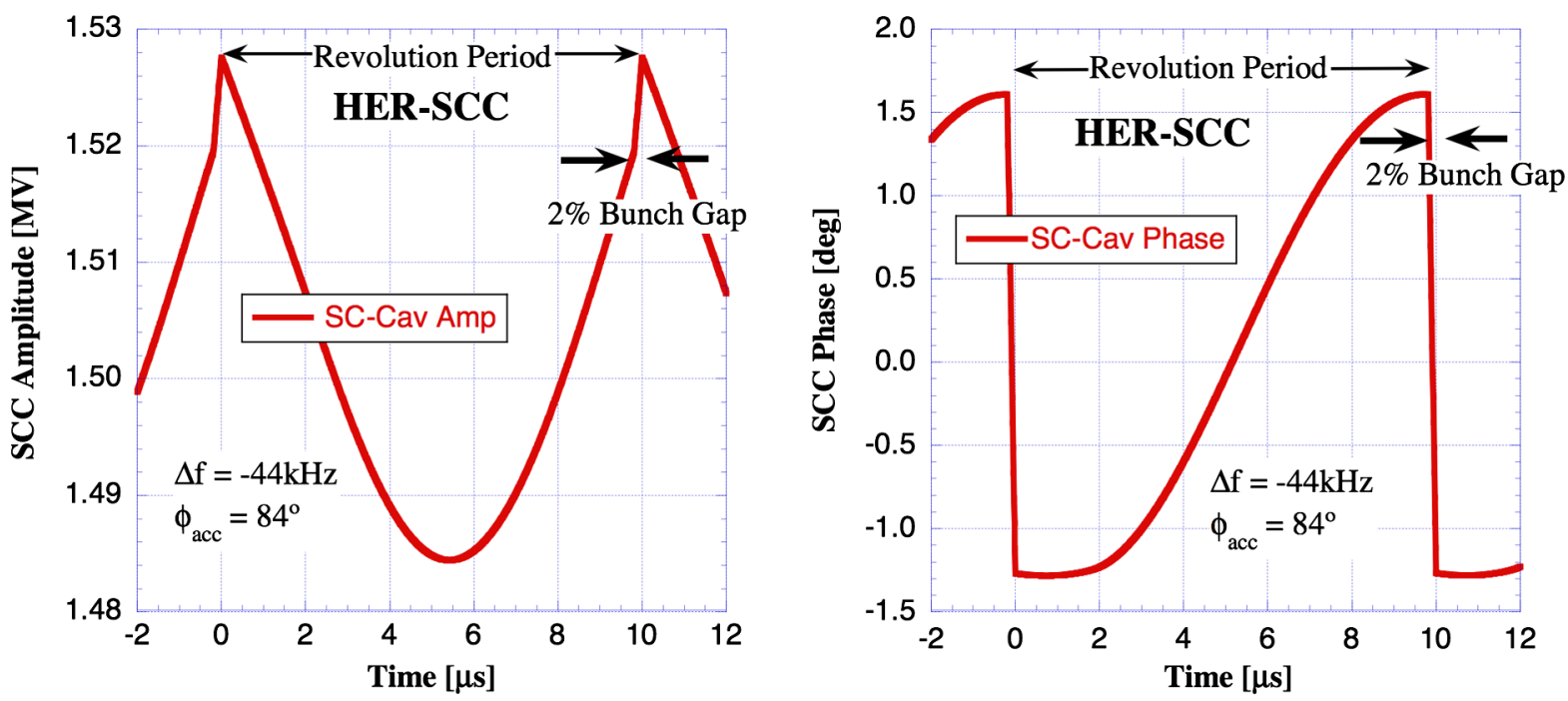

FIG. 10. SCC voltage (amplitude and phase) modulation simulated for HER in SuperKEKB.

gap transient for LER. Just as in the previous section, the revolution frequency is set to $100 \mathrm{kHz}$ instead of $99.4 \mathrm{kHz}$ to simplify the abscissa axis of time. In the figure, the periodic interval of time 0 to $10 \mu \mathrm{s}$ corresponds to one revolution, including the $2 \%$ empty gap. The time 0 corresponds to the head bunch of the train, and the empty gap is located from 9.8 to $10 \mu$ s. Figure 8 shows a plot of Fig. 7 zoomed in around the empty gap. A rapid phase change with a ringing of 6 degrees (pk-pk), following the gap, is clearly found in Figs. 7 and 8. As concluded in the previous section, the rapid phase change with ringing is attributed to the parasitic modes of ARES. In Fig. 7, except the leading part, a phase modulation of about 2 degrees due to the bunch gap transient is found along the train, which agrees with the simple analytical estimate from Eq. (1). As mentioned previously, the feedback control for the cavity voltage regulation has little effect on the rapid phase modulation in the leading part of the train because of the bandwidth of the limited closed loop, while the regulation control acts to keep the average value of $\mathbf{V}_{c}$ along the train.

Figures 9 and 10 show the simulation results of ARES and SCC, respectively, for HER. As shown in Fig. 9, a phase ringing after the gap is found in the case of ARES, just as for LER. On the other hand, in the case of SCC shown in Fig. 10, the phase ringing is not found because the SCCs are single cell cavities. Figure 11 shows the vectorsum phase of the eight ARES cavities and eight SCCs with a relative phase difference of 21.5 degrees between ARES and SCC. The total phase modulation in HER is estimated to be 2.5 degrees excluding the phase ringing at the leading part of the bunch train. 


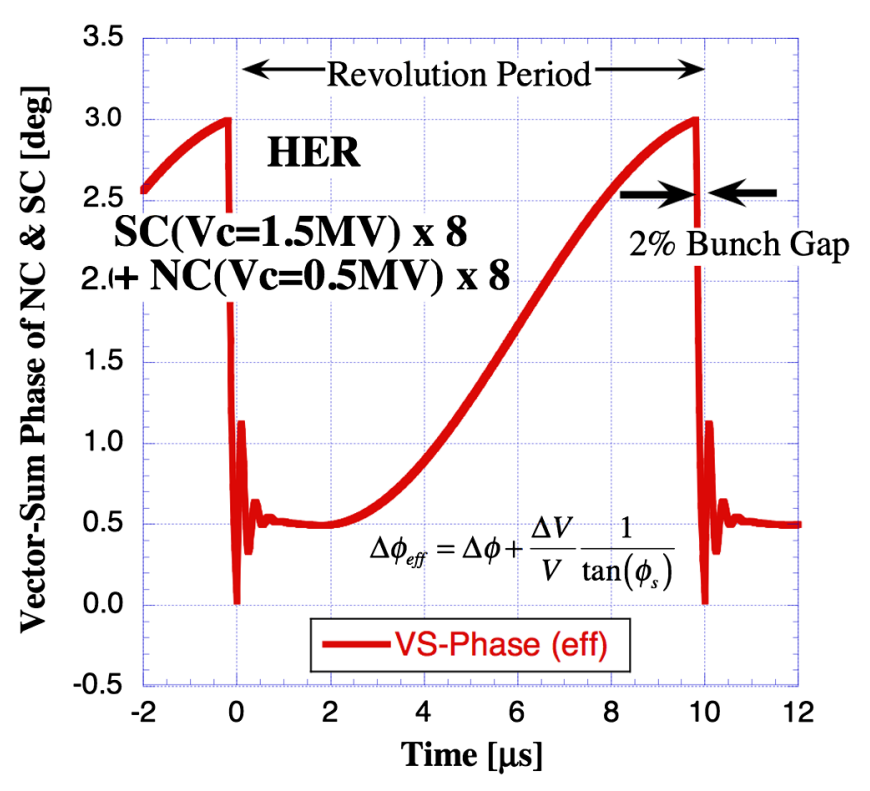

FIG. 11. Phase modulation of the vector sum of ARES and SCC voltage simulated for HER.

From the simulation results shown in Figs. 7-11, the rapid phase change in the leading part of the bunch train due to the bunch gap will be significantly larger in SuperKEKB. Figure 12 shows the phase difference between LER and HER $\left(\Delta \phi_{\mathrm{HER}}-\Delta \phi_{\mathrm{LER}}\right)$, obtained from Figs. 7 and 11. In the figure, the solid red line indicates the relative phase. A plot zoomed in around the gap is presented in the right side plot of Fig. 12. As seen in the figure, the maximum phase difference will be 5.5 degrees at the leading bunches. Besides the leading bunches, the phase difference along the train is not so large. The relative phase shift at the interaction point (IP) will be

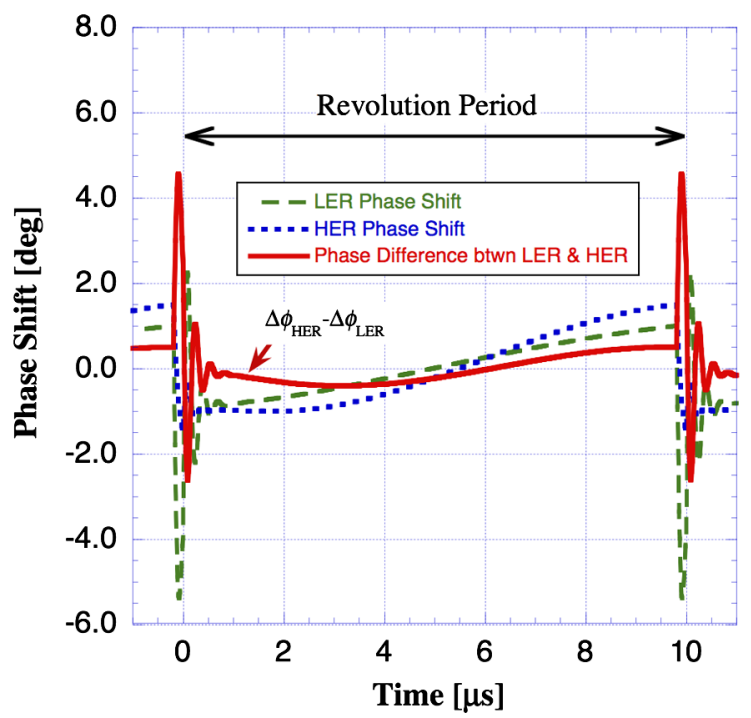

$\pm\left(\Delta \phi_{\mathrm{HER}}-\Delta \phi_{\mathrm{LER}}\right) / 4= \pm(2.5-2.0) / 4= \pm 0.125$ degrees, excluding the leading part of the bunch train. The 0.125-degree phase shift corresponds to a longitudinal displacement of $0.04 \sigma_{z}$ at the IP, where the bunch length $\left(\sigma_{z}\right)$ is $5 \mathrm{~mm}(\mathrm{rms})$.

\section{MITIGATION OF THE RELATIVE PHASE DIFFERENCE BETWEEN THE TWO RINGS FOR SUPERKEKB}

In the KEKB operation, no degradation was observed of the luminosity due to the bunch gap transient. However, in the SuperKEKB operation, the beam phase difference at the leading part of the train due to the gap transient will become much larger than that of KEKB, as presented in the previous section. Because the crossing angle between the two beams at the collision point is larger in SuperKEKB and the vertical beta function $\left(\beta_{y}{ }^{*}\right)$ is much smaller than for KEKB, the effect of the large phase difference at the leading part of the train of the colliding beams might be a crucial issue to achieve high luminosity. Beam blowup or even beam loss might happen in the worst-case scenario. In the future, a detail study of this effect from the point of view of beam-beam interactions is needed for the SuperKEKB operation. We also propose measures to mitigate the phase difference between the colliding beams as a cure if the beam-beam interaction effect turns out to be critical.

\section{A. Gap delay for the mitigation of the phase difference}

The first mitigation method is simply making a delay of the HER gap timing with respect to the LER gap at the cost of a reduced number of colliding bunches. With this method, the collision is avoided for the leading part of the LER train that has the large rapid phase change.

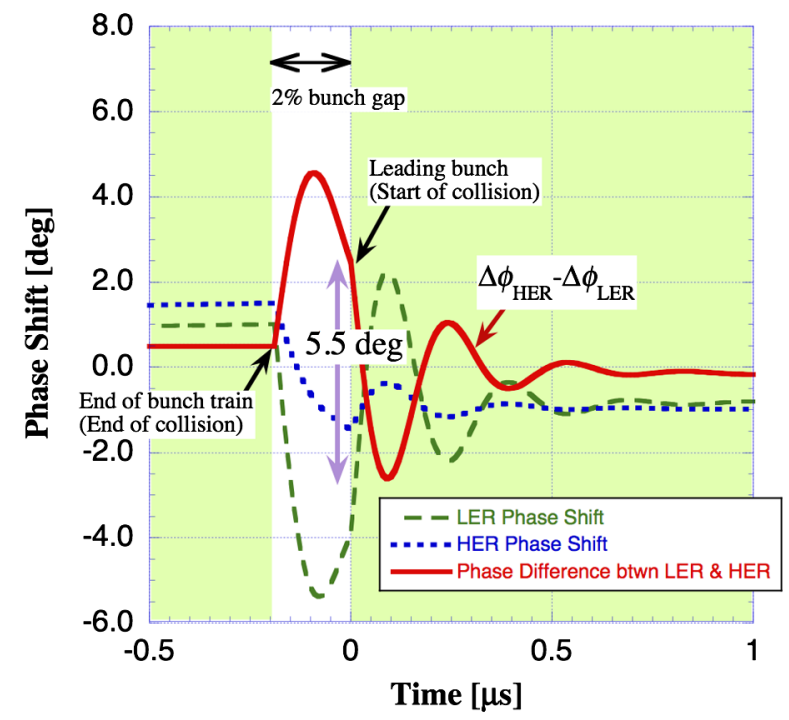

FIG. 12. Plot of the phase difference between LER and HER (red solid line $=\Delta \phi_{\mathrm{HER}}-\Delta \phi_{\mathrm{LER}}$ ). The right side is zoomed in around the gap. 


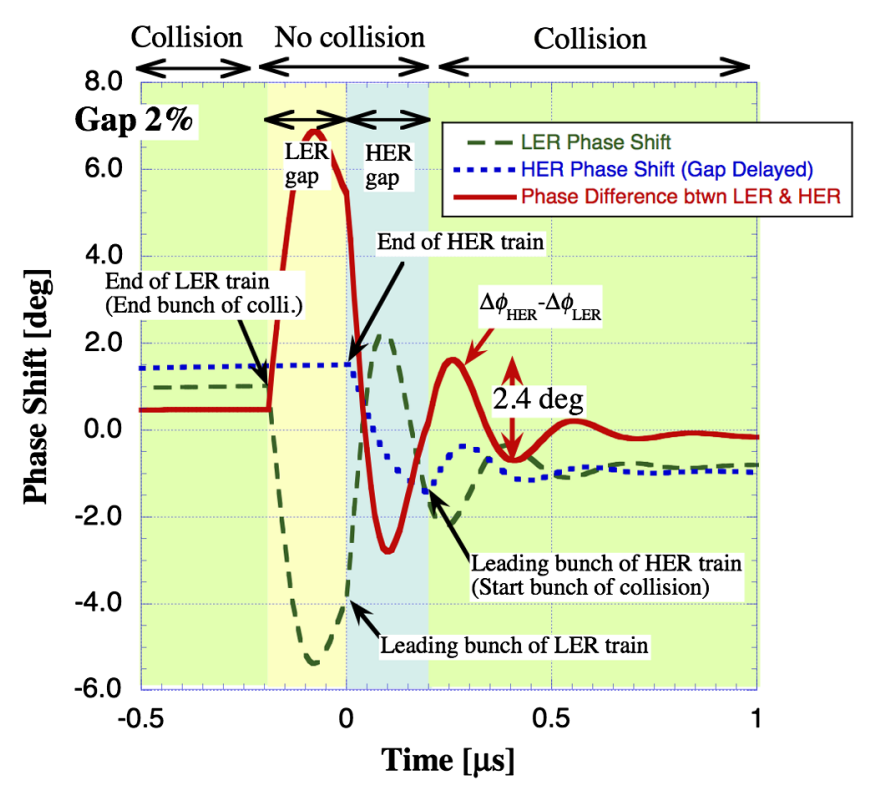

FIG. 13. Phase difference between LER and HER (red solid line) for a HER gap delay of $200 \mathrm{~ns}$. The phase difference at the leading part of the train is reduced to 2.4 degrees for the collision.

Figure 13 shows the phase difference when the HER gap is being delayed by a gap length of $200 \mathrm{~ns}$. In this case, the phase difference between the two beams is reduced to 2.4 degrees at the leading part of the colliding bunches, while the phase difference in the other part of the train along the revolution period is similar to the case of no delay. As another example, we evaluated a 3\% gap in both rings with the HER gap delayed by a gap length of $300 \mathrm{~ns}$. Here, the same bunch current is assumed as for the 2\% gap. The result is shown in Fig. 14. In this case, the phase difference between the two beams almost cancels at the leading part of the train (Fig. 14, left). However, the phase difference along the entire bunch train in the revolution period is increased to 1.6 degrees (Fig. 14, right), which comes from the increased gap length, as envisaged from Eq. (1). These results are summarized in Table V. The number of colliding bunches is reduced by $2 \%$ (2\% gap with 200 -ns delay) or $4 \%$ (3\% gap with 300 -ns delay) compared to the original fill pattern ( $2 \%$ gap, no delay). Although the reduced number of colliding bunches results in a luminosity reduction by the same amount, the possible disaster of beam performance deterioration due to an aberrant beam-beam interaction caused by the large relative phase difference in the colliding bunches could be avoided with this method.

\section{B. Varying bunch currents at the leading bunches with gap delay}

For further mitigation, we also investigated a measurement with a changing bunch fill pattern. We assume that the bunch current in the main part of the train is set at the maximum allowed bunch current from the viewpoints of optics, dynamic aperture, beam-beam, etc., and that no bunch current can exceed this value. Then, in the following, we consider only the reduction of the bunch current from this nominal (=maximum allowed) value. By appropriately adjusting the bunch current at the leading part of the train, the ringing behavior in the rapid phase change can be mitigated. For example, by gradually increasing the bunch current as a function of bunch number from the start bunch up to a few cycles of the ringing period ( $300 \mathrm{~ns})$ we could reduce the ringing magnitude. Various fill patterns including the above one can be attempted to search for the best

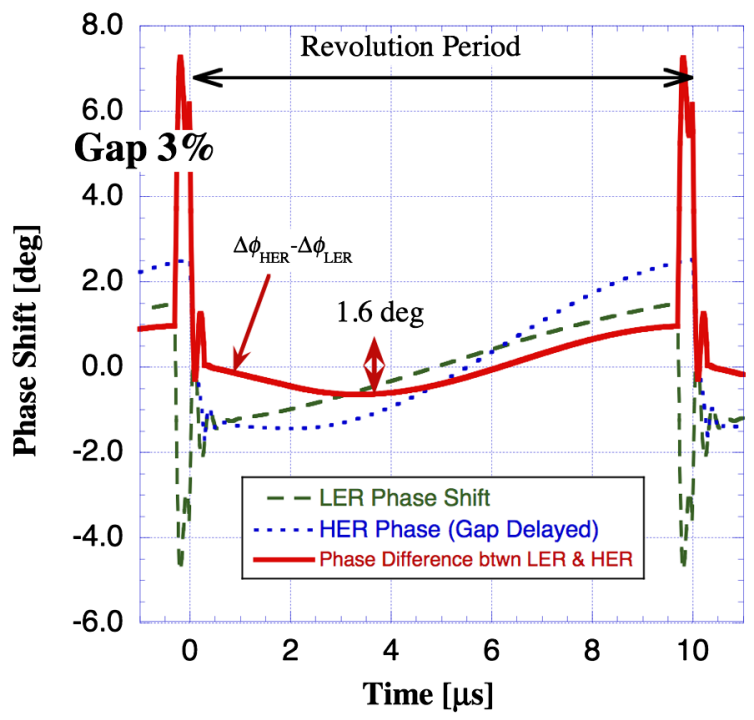

FIG. 14. Phase difference for a 3\% gap with HER gap delay. The phase difference at the leading part of the train is almost canceled, while the phase difference of the other part in the train increased to 1.6 degrees. 
TABLE V. Effects of the proposed mitigation methods on reducing the phase difference between colliding beams.

\begin{tabular}{|c|c|c|c|c|c|c|}
\hline \multirow[b]{2}{*}{ Method } & \multirow{2}{*}{$\begin{array}{l}\text { Bunch } \\
\text { gap }\end{array}$} & \multirow{2}{*}{$\begin{array}{l}\text { HER } \\
\text { delay }\end{array}$} & \multicolumn{2}{|c|}{$\begin{array}{c}\text { Phase difference } \\
\left(\left|\Delta \phi_{\mathrm{HER}}-\Delta \phi_{\mathrm{LER}}\right|\right)[\text { degrees }] \\
\end{array}$} & \multirow{2}{*}{$\begin{array}{c}\text { Longitudinal } \\
\text { displacement } \\
@ \operatorname{IP}\left(\sigma_{z}=5 \mathrm{~mm}\right)\end{array}$} & \multirow{2}{*}{$\begin{array}{l}\text { Rate of num. of } \\
\text { colliding bunche }\end{array}$} \\
\hline & & & Leading part & The rest of train & & \\
\hline \multirow{3}{*}{ HER gap delay } & $2 \%$ & no delay & 5.5 & 0.9 & $0.44 \sigma_{z}$ & \\
\hline & $2 \%$ & $2 \%(200 \mathrm{~ns})$ & 2.4 & 0.9 & $0.19 \sigma_{z}$ & $-2 \%$ \\
\hline & $3 \%$ & 3\% (300 ns) & $<0.2$ & 1.6 & $0.13 \sigma_{z}$ & $-4 \%$ \\
\hline LER 2 steps +HER gap delay & $2 \%$ & $160 \mathrm{~ns}$ & 0.4 & 0.5 & $0.07 \sigma_{z}$ & $-1.6 \%$ \\
\hline
\end{tabular}

result. From the viewpoint of practical beam operation, however, a simpler fill pattern would be favored. Consequently, we investigate below the case of a simple fill pattern with only two steps of bunch current increase at the

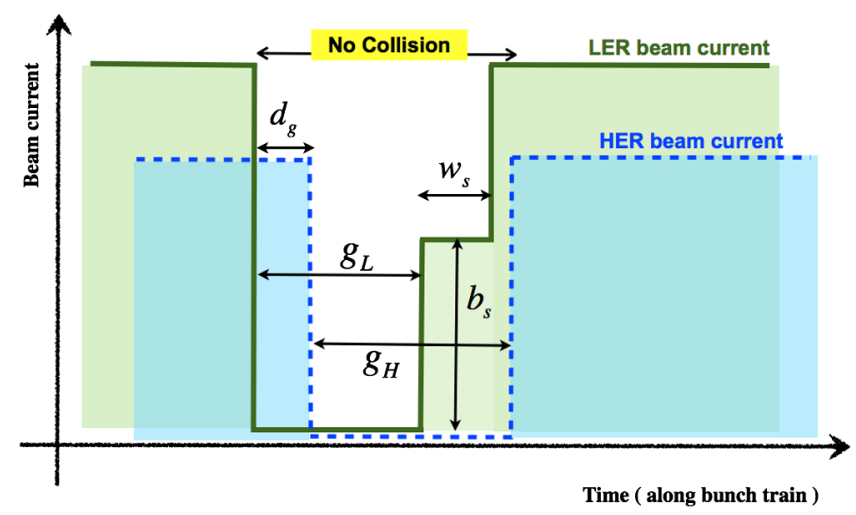

FIG. 15. Illustration of a bunch fill pattern for more effective mitigation. The bunch current at the leading part of the LER train is increased in two steps with a time interval $w_{\mathrm{s}}$ and the bunch current at the first step by $b_{s}$. The gap lengths are $\mathrm{g}_{L}$ and $\mathrm{g}_{H}$ for LER and HER, respectively, and the HER gap is delayed by $d_{g}$. leading part of the LER train combined with the HER gap delay.

Figure 15 shows the fill pattern considered here. The bunch current at the leading part of the LER train is increased in two steps with a time interval $w_{s}$ and the first step bunch current increase of $b_{s}$. The gap lengths are $g_{L}$ and $g_{H}$ for LER and HER, respectively, and the HER gap is delayed by $d_{g}$. The gap lengths are set to $2 \%$ $\left(g_{L}=g_{H}=200 \mathrm{~ns}\right)$, which is the minimum length required from the rise time of the abort kicker. For simplicity, $b_{s}$ is set to half of the nominal bunch current. The simulations were performed by changing the parameters $w_{s}$ and $d_{g}$ with a step of $10 \mathrm{~ns}$. The following guidelines were used: (1) the phase changes due to the first and second step should mostly cancel each other after the second step ( $w_{s}$ should be about half of the ringing period), and (2) the LER phase ringing after the second step should be synchronized with the HER phase change at the leading part of the HER train. The best result was obtained with 140 and $160 \mathrm{~ns}$ for $w_{s}$ and $d_{g}$, respectively. The result is shown in Fig. 16. The phase difference between LER and HER is significantly reduced to 0.4 degrees at the leading part of
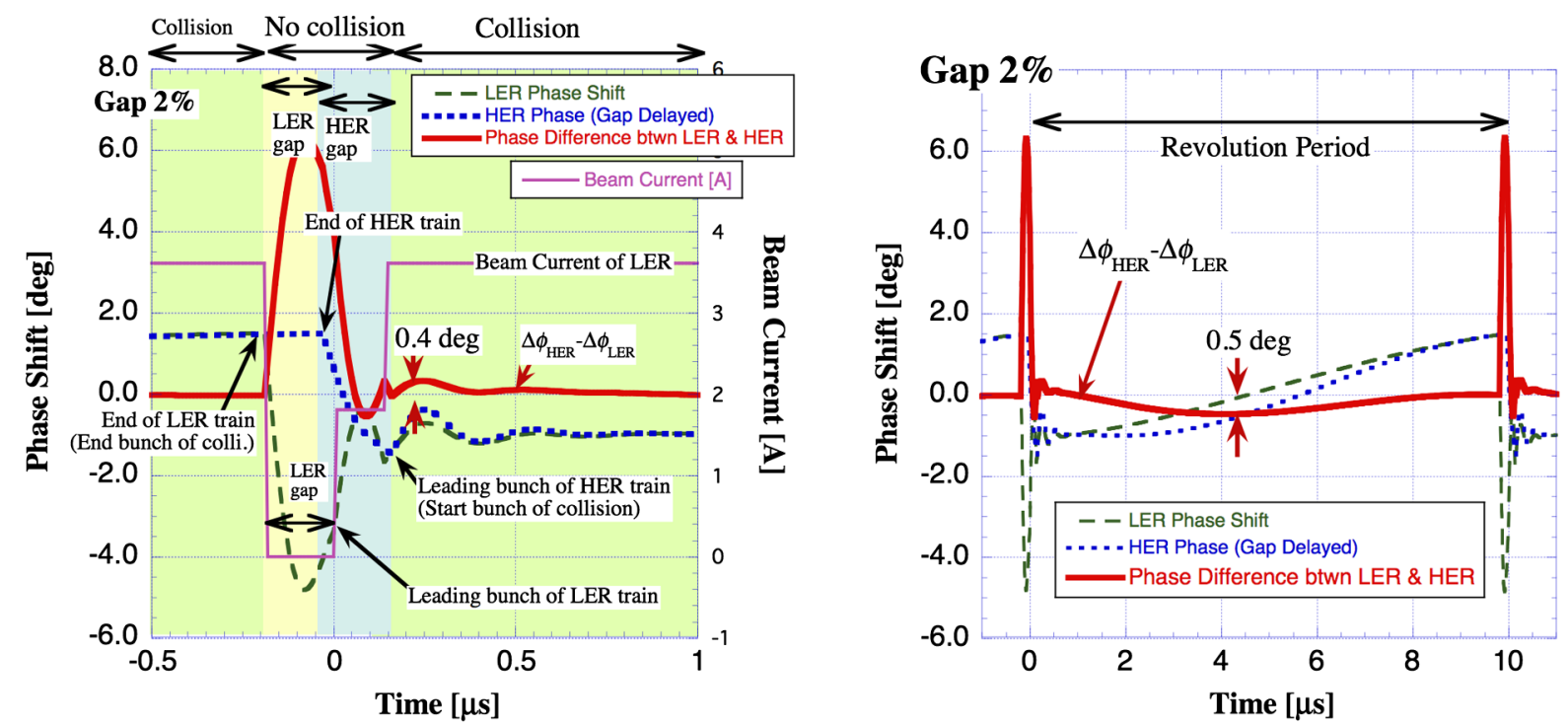

FIG. 16. Simulation result of phase difference for the best case of the fill pattern shown in Fig. 15. The gap length of both rings is $2 \%$ (200 ns), the current height at the initial step of the LER leading part $\left(b_{s}\right)$ is half of the nominal current, the length of the initial step ( $\left.w_{s}\right)$ is $140 \mathrm{~ns}$ and the delay of the HER gap $\left(d_{g}\right)$ is $160 \mathrm{~ns}$. 
the collision (Fig. 16, left), while the entire phase difference along the bunch train is kept sufficiently small (Fig. 16, right). The result is also shown in Table V. Thus, we have demonstrated that a simple bunch fill pattern change with only two steps in LER combined with a HER gap delay further reduces the phase difference between the two beams. This method gives a more effective mitigation compared with only the HER gap delay cases.

It should be noted that the best optimization for the bunch pattern strongly depends on the operation conditions such as beam optics, dynamic aperture, beam-beam effects, and so on. These operation conditions will be optimized in future SuperKEKB operation. The best optimization for the fill pattern and gap delay will be investigated based on the best operation conditions.

\section{CONCLUSION}

We have presented an investigation of the bunch gap transient effect using an advanced transient simulation, which treats transient loading in the three-cavity system of the ARES cavities. The result clarifies that the rapid phase change at the leading part of the bunch train observed in the KEKB operation is attributed to the parasitic modes ( 0 and $\pi$-modes) excitation in the ARES cavities. The phase change and ringing characteristics depend significantly on the property of the coupling cavity of ARES.

The bunch gap transient effect in SuperKEKB was also evaluated using the new simulation. The result showed that a large phase change with a ringing of about 6 degrees will arise at the leading part of the bunch train in LER with twice as high stored beam current as that achieved in KEKB. If the beam-beam interaction effect due to the relative phase difference in LER and HER turns out to be a critical issue for SuperKEKB with a very small $\beta_{y}{ }^{*}$, we propose methods of mitigation to reduce the relative phase difference. As one of the methods, we show that a simple bunch fill pattern change, with only two steps in the LER leading part combined with the HER gap delay, significantly reduces the phase difference between the two beams.

We believe that analyzing the interaction of the rf system with the beam using the new simulation is useful for increasing the luminosity of SuperKEKB, a luminosity frontier collider.

[1] T. Abe et al., KEKB accelerator, Prog. Theor. Exp. Phys. 2013, 30001 (2013).

[2] P. B. Wilson, Report No. PEP-Note 37, SPEAR-163, 1973.

[3] K. Akai et al., RF systems for the KEK B-Factory, Nucl. Instrum. Methods Phys. Res., Sect. A 499, 45 (2003).

[4] T. Abe et al., Performance and operation results of the rf systems at the KEK B-Factory, Prog. Theor. Exp. Phys. 2013, 03A006 (2013).
[5] Y. Yamazaki and T. Kageyama, A three-cavity system which suppresses the coupled-bunch instability associated with the accelerating mode, Part. Accel. 44, 107 (1994).

[6] T. Kageyama, Y. Takeuchi, N. Akasaka, F. Naito, H. Sakai, H. Mizuno, K. Akai, E. Ezura, H. Nakanishi, Y. Yamazaki, S. Miura, A. Takahashi, and M. Hamaoka, in, Proceedings of the 1st Asian Particle Accelerator Conference, Tsukuba, 1998, KEK-Proceedings-98-10, (KEK, Tsukuba, 1998), p. 773.

[7] T. Furuya, K. Akai, K. Asano, E. Ezura, K. Hara, K. Hosoyama, A. Kabe, Y. Kojima, S. Mitsunobu, Y. Morita, H. Nakanishi, T. Shishido, T. Tajima, T. Takahashi, T. Takashima, S. Yoshimoto, Y. Ishi, Y. Kijima, T. Murai, and K. Sennyu, in, Proceedings of the 5th European Particle Accelerator Conference, Barcelona, 1996 (IOP, London, 1996), p. 2121.

[8] S. Mitsunobu, K. Akai, E. Ezura, T. Furuya, K. Hara, K. Hosoyama, A. Kabe, Y. Kojima, Y. Morita, H. Nakai, and T. Tajima, Superconducting cavities for KEK B factory, Adv. Cryog. Eng. 45A, 861 (2000).

[9] T. Kageyama, T. Abe, H. Sakai, Y. Takeuchi, and K. Yoshino, in, Proceedings of the 8th annual meeting of Particle Accelerator Society of Japan, Tsukuba, 2011 (PASJ, Tokyo, 2011), p. 1245.

[10] K. Akai, in, Proceedings of the 5th European Particle Accelerator Conference, Barcelona, 1996 (IOP, London, 1996), p. 205.

[11] K. Akai, T. Ieiri, and T. Kawamoto, in Proceedings of the 19th Particle Accelerator Conference, Chicago, IL, 2001 (IEEE, Piscataway, NJ, 2001), p. 2432.

[12] T. Ieiri, K. Akai, H. Fukuma, T. Kawamoto, E. Kikutani, and M. Tobiyama, Bunch-by-bunch measurements of the betatron tune and the synchronous phase and their applications to beam dynamics at KEKB, Phys. Rev. ST Accel. Beams 5, 094402 (2002).

[13] T. Ieiri, K. Akai, H. Fukuma, and M. Tobiyama, Beam dynamics measurements using a gated beam-position monitor at KEKB, Nucl. Instrum. Methods Phys. Res., Sect. A 606, 248 (2009).

[14] H. Kichimi, K. Trabelsi, S. Uehara, M. Nakao, K. Akai, T. Ieiri, M. Tobiyama, M. D. Jones, M. W. Peters, G. S. Varner, and T. E. Browder, KEKB beam collision stability at the picosecond timing and micro position resolution as observed with the Bell Detector, J. Instrum. 5 P03011 (2010).

[15] Y. Ohnishi et al., Accelerator design at SuperKEKB, Prog. Theor. Exp. Phys. 2013, 3A011 (2013).

[16] T. Abe et al., Belle II Technical Design Report, KEK Report 2010-1, 2010.

[17] K. Akai, T. Abe, K. Ebihara, M. Ono, T. Kageyama, A. Kabe, H. Sakai, S. Takano, Y. Takeuchi, K. Nakanishi, M. Nishiwaki, T. Furuya, K. Marutsuka, S. Mitsunobu, Y. Morita, M. Yoshida, K. Yoshino, and S. Yoshimoto, in, Proceedings of the 7th annual meeting of Particle Accelerator Society of Japan, Himeji, 2010 (PASJ, Tokyo, 2010), p. 1232.

[18] T. Kobayashi, K. Akai, K. Ebihara, A. Kabe, K. Nakanishi, M. Nishiwaki, J. Odagiri, H. Deguchi, K. Hayashi, T. Iwaki, and M. Ryoshi, in, Proceedings of the 5th International Particle Accelerator Conference, Dresden, 2014 (JACoW, 2014), p. 2444. 
[19] E. Ezura, S. Yoshimoto, and K. Akai, in, Proceedings of the International Workshop on Collective Effects and Impedance for B Factories, Tsukuba, 1995, KEK-Proceedings96-6 (KEK, Tsukuba, 1995), p. 437.

[20] K. Akai, N. Akasaka, K. Ebihara, E. Ezura, M. Suetake, and S. Yoshimoto, in Proceedings of the 6th European Particle Accelerator Conference, Stockholm, 1998 (IOP, London, 1998), p. 1749.

[21] D. Boussard, in Proceedings of the 1991 Particle Accelerator Conference, San Francisco, CA, 1991 (IEEE, New York, 1991), p. 2447.

[22] K. Akai and E. Ezura, in Proceedings of the Fourth European Particle Accelerator Conference EPAC 94,
London, England (World Scientific, River Edge, NJ, 1994), p. 1141.

[23] KEKB Design Report, KEK Report No. 95-7, 1995.

[24] T. Schilcher, Ph.D. Thesis, Universität Hamburg, 1998.

[25] T. Abe, in, Proc. of 30th Advanced ICFA Beam Dynamics Workshop on High Luminosity $e^{+} e^{-}$Collisions, Stanford, 2003 (SLAC, Stanford, 2003), p. WGB03.

[26] T. Kobayashi, K. Akai, K. Ebihara, J. Odagiri, A. Kabe, K. Nakanishi, M. Nishiwaki, H. Deguchi, K. Harumatsu, K. Hayashi, T. Iwaki, J. Mizuno, J. Nishio, and M. Ryoshi, in Proceedings of the 4th International Particle Accelerator Conference, IPAC-2013, Shanghai, China, 2013 (JACoW, Shanghai, China, 2013), p. 2953. 